\title{
Geochemical characterization of fluoride in water, table salt, active sediment, rock and soil samples and its possible relationship with the prevalence of enamel fluorosis in children in four municipalities of the department of Huila (Colombia)
}

Stefania Martignon \& Mario Omar Opazo-Gutiérrez \& Möritz Velásquez-Riaño \& Iván Rodrigo OrjuelaOsorio \& Viviana Avila \& Esperanza Angeles Martinez-Mier \& María Clara González-Carrera \& Jaime Alberto Ruiz-Carrizosa \& Blanca Cecilia Silva-Hermida

\begin{abstract}
Fluoride is an element that affects teeth and bone formation in animals and humans. Though the use of systemic fluoride is an evidence-based caries preventive measure, excessive ingestion can impair tooth development, mainly the mineralization of tooth enamel, leading to a condition known as enamel fluorosis. In this study, we investigated the geochemical characterization of fluoride in water, table salt, active sediment, rock and soil samples in four endemic enamel fluorosis sentinel municipalities of the department of Huila, Colombia (Pitalito, Altamira, El Agrado and Rivera) and its possible relationship with the prevalence of enamel fluorosis in children. The concentration of fluoride in drinking water, table salt, active sediment, rock and soil was evaluated by means of an ion selective electrode and the geochemical analyses were performed using X-Ray fluorescence. Geochemical analysis revealed fluoride concentrations under $15 \mathrm{mg} / \mathrm{kg}$ in active sediment, rock and soil samples, not indicative of a significant delivery to the watersheds studied. The concentration of fluoride in table salt was found to be under the inferior limit (less than $180 \mu \mathrm{g} / \mathrm{g}$ ) established by the Colombian regulations. Likewise, exposure doses for fluoride water intake did not exceed the recommended total dose for all ages from 6 months. Although the evidence does not point out at rocks, soils, fluoride-bearing minerals, fluoridated salt and water, the hypothesis of these elements as responsible of the current prevalence of enamel fluorosis cannot be discarded since, aqueducts might have undergone significant changes overtime.
\end{abstract}

Keywords: fluoride, water, salt, geochemistry, enamel fluorosis.

\footnotetext{
Acknowledgments

This study was conducted with the financial support of COLCIENCIAS, Colombia Contract 442-2012. The authors thank Universidad El Bosque, the Secretary of Health of the department of Huila and the local governments of the municipalities of Altamira, El Agrado, Pitalito and Rivera for their support in the development of this study.
} 
S. Martignon (*) . V. Avila . J. A. Ruiz-Carrizosa

UNICA - Caries Research Unit, Research Vice-rectory, Universidad El Bosque, Av. Cra. 9 No. 131A-02, Edificio Fundadores, $2^{\circ}$ piso, Bogotá 110121, Colombia

e-mail: martignonstefania@unbosque.edu.co

S. Martignon

Dental Innovation and Translation Centre, King's College Dental Institute, 17 Tower Wing Room 37, Great Maze Pound, London SE1 9RT, UK

M. O. Opazo-Gutiérrez . I. R. Orjuela-Osorio

Grupo Agua, Salud y Ambiente, Faculty of Engineering, Universidad El Bosque, Av. Cra. 9 No. 131A-02, Bogotá 110121, Colombia

M. Velásquez-Riaño

Grupo Producción Limpia - Choc Izone, Faculty of Engineering, Universidad El Bosque, Av. Cra. 9 No. 131A-02, Bogotá 110121, Colombia

\section{E. A. Martinez-Mier}

Department of Cariology, Operative Dentistry and Dental Public Health, Indiana University School of Dentistry, 415 Lansing Street, Indianapolis, IN 46202-2876, USA

M. C. González-Carrera

UMIMC - Unidad de Manejo Integral de MalformacionesCraneofaciales, Dental Scool, Universidad El Bosque, Av. Cra. 9 No. 131A-02, Bogotá 110121, Colombia

B. C. Silva-Hermida

Department Secretary of Health - Huila, Cra. 20 No. 5B, -36 Neiva, Huila, Colombia 


\section{Introduction}

In Colombia the monitoring of fluoride concentrations in water is performed by sentinel surveillance. During one monitoring period (2012-2014) there was evidence of fluoride levels above $1 \mathrm{mg} / \mathrm{L}$ in 13 different regions. Among those, the departments of Huila (southwest) and Casanare (east) reported concentrations between 1.10 and $3.99 \mathrm{mg} / \mathrm{L}$ of fluoride. The situation in the department of Huila and its municipalities has been cause for concern, since there are reports of both high fluoride levels in drinking water and a high prevalence of enamel fluorosis (Servicio Geológico Colombiano, 2011).

The ingestion of fluoride above "optimal” levels ( $>0.07 \mathrm{mg} \mathrm{F} / \mathrm{kg} /$ day) during tooth formation can induce a developmental defect of the enamel known as enamel fluorosis. Fluorosed enamel is more porous than sound enamel (Fejerskov et al, 1994) and there is in vitro evidence suggesting that fluorosed enamel increases the risk of developing dental caries lesions (Marin et al, 2016). Furthermore, enamel fluorosis is considered as a public health problem, with biological, aesthetic and clinical consequences for the individual, though no consensus has been reached among the scientific community (Fejerskov et al, 1994; Suma et al, 2008).

The department of Huila is in the southwest part of the country, in the middle of the central and east branches of the Colombian Andes. It has latitude varying from 01³0’04', N (La Fragua Peaks) to 035’12'’ N (Riachón River headwaters) and from $74^{\circ} 25^{\prime} 24^{\prime}$ ' W (Las Oseras peak) to $76^{\circ} 35^{\prime} 16^{\prime}$ ' W (Las Papas alpine tundra). The department has an extension of $19.890 \mathrm{Km}^{2}, 1.74 \%$ of Colombia's total extension. It also has a high geo-diversity, given the origin of the Andean branches: volcanic for the central branch with igneous rocks predominance; tectonic for the east branch with sedimentary rocks predominance. Three different physiographic regions can be distinguished as one) the Central Branch and the Colombian Massif, which joints the eastern foothills and the central region of the Department with the mountainous area of Las Minas; 2) the Magdalena Valley, which divides the department from southeast to northeast and 3) the East Branch, which includes the West Foothills and the Garzón Massif (IGAC, 2008).

In nature, fluorine occurs in interstratified pure minerals or as an impurity in other minerals. Fluorite, apatite, cryolite and lepidolite are fluorine-rich minerals. Other fluorine-bearing minerals are basalt, andesite, fluorite, gabbro, diabase, dolomite and argillite (Frankenberg et al, 1996). Fluorine associates with other elements, in particular geological environments. There are four major deposits of fluorine in nature: 1) igneous rocks (disseminated deposits, pegmatite deposits, carbonatite 
and contact aureole deposits); 2) sedimentary rocks (lacustrine deposits, evaporite, marine carbonates and phosphate rock); 3) regional metamorphic rocks, and 4) hydrothermal fluorite deposits (deposits in veins, mantles, trellises and alteration zones) (Clavijo and Ballesteros, 1987). These nature deposits can be measured in rock, soil and active sediment samples. Active sediment corresponds to sediments that have been dragged off from high parts of the mountains to the riverbeds by water runoff and are deposited at the edge of the tributaries. In the department of Huila pure fluorine-bearing minerals have been found in different environments (Velandia et al, 2001).

The Pan-American Health Organization (PAHO) recommends the performance of epidemiological sentinel surveillance (OPS/OMS, 1995). In the department of Huila there is a lack of consistency between fluoride concentrations in watersheds, drinking water, table salt and the prevalence of enamel fluorosis. In this case, sentinel surveillance might help in the determination of the relationship between enamel fluorosis, caries, and fluoride in the environment.

The aim of this study was to perform geochemical characterization of fluoride in water, table salt, active sediment, rock and soil samples in four endemic enamel fluorosis sentinel municipalities of the department of Huila, in Colombia (Pitalito, Altamira, El Agrado and Rivera). Furthermore, we evaluated the possible relationship between fluoride content in these sources and the prevalence of the enamel-developed defect enamel fluorosis, in children from those communities.

\section{Material and methods}

This study is part of the multi-disciplinary project entitled "Model of environmental, epidemiological, clinical, histological and inorganic characterization of enamel fluorosis in Children” with Ethical Board approval from Universidad El Bosque (UEB 005-2012).

\section{Study Sites}

The study sites included four sentinel municipalities in the Department of Huila (Colombia) Pitalito, Altamira, El Agrado and Rivera, selected based on documented enamel fluorosis prevalence in children (Jiménez et al, 2006; Montaña-Mesa, 2008; Tellez et al, 2012). The municipality of Pitalito is located in the south of Huila, has an altitude of $1300 \mathrm{~m}$ asl, average temperature of $24^{\circ} \mathrm{C}$, an extension of $666 \mathrm{Km}^{2}$ and approximately 109 thousand inhabitants, $60 \%$ of them living in the urban areas (Alcaldía de Pitalito, 2016). The municipalities of 
Altamira, El Agrado and Rivera are located in the center of the department. Altamira has an altitude of $1079 \mathrm{~m}$ asl, average temperature of $23^{\circ} \mathrm{C}$ and an extension of $118.18 \mathrm{Km}^{2}$, with approximately three thousand six hundred inhabitants (Alcaldía de Altamira, 2016). The municipality of El Agrado has an altitude of $907 \mathrm{~m}$ asl, an average temperature of $24^{\circ} \mathrm{C}$ and eight thousand five hundred inhabitants (Alcaldía de El Agrado, 2016). The municipality of Rivera is also located in the center, but in northeast direction, at $700 \mathrm{~m}$ asl with three varieties of climate: warm, temperate and cold. Its extension is $435 \mathrm{Km}^{2}$ and the estimated population is fourteen thousand one hundred inhabitants (Alcaldía de Rivera, 2016) (Figure 1).

\section{Geochemical characterization of fluoride}

We followed the protocols reported by Zinck (2012), the Colombian Geology and Mining Institute (Velandia et al, 2001; Rojas et al, 2010) and the Agustín Coddazi Geographic Institute (IGAC, 1994) for the regional geologic recognition, geological and geomorphological characterization and field sampling, respectively. In each site, soil samples of horizons A (surface), B (subsoil) and C (substratum) were taken, as well as rocks, water and active sediment. Samples were taken by duplicate. Each sample was packed and marked as stated by the protocols mentioned above.

Guachicos river (Pitalito), Perica creek (Altamira), Mosca creek (El Agrado) and Negro river (Rivera) were identified as the main micro-basins from the water supply systems of the four sentinel municipalities. The areas were subdivided for sampling purposes, as follows (Figure 1):

- Upper basin: from the headwaters supplying the river network up to a third of the total riverbed length.

- Middle basin: sample taken from approximately the middle third of the riverbed or after encounter with a tributary along the course of the basin.

- Lower basin: before the water-treatment plant water intake.

- Additionally, water samples from the urban network's check points established by each municipality's sanitary office were taken, following the protocol established by the regulatory authorities (NTC/ISO 5667-1 ICONTEC, 1995).

Figure 1. Geographical location of sampling sites - Department of Huila Colombia.

Exploratory geochemical analyses by means of X-Ray fluorescence were 
conducted to identify major mineral groups in active sediment, rock and soil samples.

Quantification of fluoride in water, table salt, active sediment, rock and soil was performed using the fluoride-selective electrode method (Thomas et al, 1997; Martínez-Mier et al, 2009; APHA, 2012). Fluoride concentration in table salt was evaluated in 84 samples, distributed as follows: Pitalito $(n=38)$, Altamira $(n=10)$, El Agrado $(n=16)$ and Rivera $(n=20)$. Fluoride in salt and water samples was determined by duplicate in the Fluoride Laboratory of the Oral Health Research Unit (OHRI) at the Indiana University School of Dentistry in Indianapolis, IN, USA. Other samples were analyzed at the Alpha Laboratory in the city of Bogota, Colombia (NIT 900196109-1).

Determination of the prevalence of enamel fluorosis in children from the four municipalities

In total, 840 children from two age groups (3-7 and 9-14 years-old) from the four municipalities were examined using the TF Index (Thylstrup and Fejerskov, 1978). The index discriminates among absence (TFI-0) and presence of the condition (TFI-1 to TFI-9), classifying enamel fluorosis according to its severity in mild (TFI-1 to TFI-2), moderate (TFI-3 to TFI-4), and severe (TFI-5 to TFI-9) (Thylstrup and Fejerskov, 1978).

Determination of water ingestion exposure dose (IDag)

The water ingestion exposure doses (IDag) were assessed taking into account the fluoride recommended total optimal daily dose $(<0.05 \mathrm{mg} / \mathrm{kg} / \mathrm{day})$. It was calculated as follows (ATSDR, 2005):

where

$$
I D a g=\frac{C x I R \times E F}{B W}
$$

$\mathrm{C}=$ contaminant concentration $(\mathrm{mg} / \mathrm{L})$

IR = intake rate of contaminated medium (L/day) (It was worked with an standard value of $1 \mathrm{~L}$ /day for children)EF = exposure factor (It was worked with a value of 1 representing a daily exposure to the contaminant)

BW = body weight $(\mathrm{kg}$ ) (The average body weight was determined in $20 \mathrm{~kg}$ for preschoolers and $40 \mathrm{~kg}$ for schoolchildren). 


\section{Statistical analyses}

The range of water, table salt, active sediment, rock and soil fluoride concentrations was calculated for samples from each municipality. Mean and standard deviation were also calculated. Correlation analysis and ANOVA test were performed between the different age groups for exposure dose $(\mathrm{p} \leq 0.05)$.

\section{Results}

Geochemical characterization of fluoride

The fluoride analyses performed in all samples of water obtained from water tributaries (Table 1), soil, rocks, and active sediments through the fluorideselective electrode indicated that concentrations of the ion were below $15 \mathrm{mg} / \mathrm{kg}$ in the four sentinel municipalities. These results were further confirmed by the X-ray fluorescence's finding of a lack of pure fluorine minerals in the analyzed samples (active sediment, rock and soil from A, B and C horizons) (Table 2). 
Table 1. Fluoride concentration in water obtained from water tributaries.

\begin{tabular}{|c|c|c|c|c|c|c|c|c|c|c|c|c|c|c|}
\hline Municipality & \multicolumn{9}{|c|}{ Pitalito samples } & \multicolumn{3}{c|}{ Altamira samples } & \multicolumn{3}{|c|}{ El Agrado samples } \\
Characteristic
\end{tabular}


Table 2. Geochemical characterization of fluoride in active sediment, rock and soil from A, B and C horizons samples obtained from the four Huila-sentinel municipalities.

\begin{tabular}{|c|c|c|c|c|c|c|c|c|c|c|c|c|c|c|c|c|c|c|c|c|c|c|c|c|c|c|c|c|c|c|}
\hline \multirow{3}{*}{\begin{tabular}{|l|} 
Sample \\
Municipality \\
\end{tabular}} & \multicolumn{10}{|c|}{ Soil samples } & \multicolumn{9}{|c|}{ Active sediment samples } & \multicolumn{11}{|c|}{ Rock samples } \\
\hline & \multicolumn{3}{|c|}{ Pitalito } & \multicolumn{3}{|c|}{ Altamira } & \multicolumn{4}{|c|}{ Rivera } & \multicolumn{4}{|c|}{ Pitalito } & \multirow{2}{*}{\begin{tabular}{|c|} 
Altamira \\
Lower \\
basin El \\
Cedro \\
creek \\
\end{tabular}} & \multirow[b]{2}{*}{\begin{tabular}{|c|} 
Middle \\
basin EI \\
Cedro \\
creek \\
\end{tabular}} & \multirow{2}{*}{\begin{tabular}{|c|}
$\begin{array}{c}\mathrm{El} \\
\text { Agrado }\end{array}$ \\
El \\
Pescado \\
creek
\end{tabular}} & \multicolumn{2}{|c|}{ Rivera } & \multicolumn{3}{|c|}{ Pitalito } & \multicolumn{3}{|c|}{ Altamira } & \multicolumn{3}{|c|}{ El Agrado } & \multirow{2}{*}{\begin{tabular}{|l|} 
Rivera \\
Upper \\
basin \\
Negro \\
river \\
\end{tabular}} & \multirow[b]{2}{*}{$\begin{array}{l}\text { Lowe } \\
\text { bassir } \\
\text { Negr } \\
\text { river }\end{array}$} \\
\hline & $\mid \begin{array}{c}\text { El Cedro } \\
\text { creek }\end{array}$ & $\begin{array}{c}\text { El Cedro } \\
\text { creek }\end{array}$ & \begin{tabular}{|c|} 
Lower \\
basin Gua- \\
chicos- \\
river \\
\end{tabular} & \begin{tabular}{|c|} 
Lower \\
basin EI \\
Cedro \\
creek \\
\end{tabular} & \begin{tabular}{|c|} 
Middle \\
basin El \\
Cedro \\
creek \\
\end{tabular} & \begin{tabular}{|c|} 
Middle \\
basin El \\
Cedro \\
creek \\
\end{tabular} & \begin{tabular}{|c|} 
Upper \\
basin \\
Negro \\
river \\
\end{tabular} & \begin{tabular}{|c|} 
Lower \\
bassin \\
Negro \\
river
\end{tabular} & \begin{tabular}{|c|} 
Lower \\
bassin \\
Negro \\
river \\
\end{tabular} & $\begin{array}{c}\text { Upper } \\
\text { basin } \\
\text { Negro } \\
\text { river } \\
\end{array}$ & $\begin{array}{c}\text { El Cedro } \\
\text { creek }\end{array}$ & \begin{tabular}{|l} 
Lower \\
basin \\
Gua- \\
chicos \\
creek \\
\end{tabular} & $\begin{array}{c}\text { ILwer } \\
\text { basin } \\
\text { Gua- } \\
\text { chicos } \\
\text { creek } \\
\end{array}$ & $\begin{array}{l}\text { Lower } \\
\text { basin } \\
\text { Gua- } \\
\text { chicos } \\
\text { river }\end{array}$ & & & & $\begin{array}{c}\text { Upper } \\
\text { basin } \\
\text { Negro } \\
\text { river }\end{array}$ & $\begin{array}{c}\text { Middle } \\
\text { basin } \\
\text { Negro } \\
\text { river } \\
\end{array}$ & \begin{tabular}{|c|} 
Gua- \\
chicos \\
creek \\
\end{tabular} & \begin{tabular}{|l|} 
Lower \\
basin \\
Gua- \\
chicos \\
river \\
\end{tabular} & $\begin{array}{c}\text { El Cedro } \\
\text { creek }\end{array}$ & \begin{tabular}{|c|} 
Middle \\
basin El \\
Cedro \\
creek \\
\end{tabular} & \begin{tabular}{|c|} 
Upper \\
basin La \\
Perica \\
creek \\
\end{tabular} & \begin{tabular}{|c|} 
Lower \\
basin EI \\
Cedro \\
creek
\end{tabular} & \begin{tabular}{|c|} 
La \\
Mosca \\
creek
\end{tabular} & $\begin{array}{c}\text { El } \\
\text { Agrado } \\
\text { creek }\end{array}$ & $\begin{array}{c}\text { El } \\
\text { Pescado } \\
\text { creek }\end{array}$ & & \\
\hline Latitude & 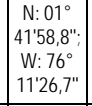 & \begin{tabular}{|} 
N: $: 01^{\circ}$ \\
$41^{\prime} 58,8^{\prime \prime}$ \\
W: \\
$11267^{\circ}$ \\
$11^{\prime \prime} 26,7^{\prime \prime}$
\end{tabular} & \begin{tabular}{|c|} 
N: $01^{\circ}$ \\
$47^{\prime} 23,2^{\prime \prime}$ \\
W: \\
: $6^{\prime} 0^{\circ}$ \\
$09^{\prime} 17,0^{\prime \prime}$ \\
\end{tabular} & \begin{tabular}{|c|} 
N: $: 01^{\circ}$ \\
$56 ' 24,8^{\prime \prime}$ \\
W: $75^{\circ}$ \\
$42^{\circ} 45,4^{\prime \prime}$ \\
\end{tabular} & $\begin{array}{c}\text { N: } 01^{\circ} \\
56^{\prime} 06,0^{\prime \prime} ; \\
\text { W: }: 5^{\circ} \\
42^{\prime} 47,1^{\prime \prime} \\
\end{array}$ & $\begin{array}{c}\text { N: } 01^{\circ} \\
566^{\circ} 06,0^{\prime \prime} \\
\text { W: } 75^{\circ} \\
42^{2} 47,1^{\prime \prime} \\
\end{array}$ & \begin{tabular}{|} 
N: $02^{\circ}$ \\
$4447,9^{\prime \prime}$ \\
W: $5^{\circ}$ \\
$1237,1^{\prime \prime}$
\end{tabular} & INA & INA & $\begin{array}{c}\text { N: } 02^{\circ} \\
44^{\circ} 47,9^{\prime \prime} \\
\text { W: }: 5^{\circ} \\
12^{\prime} 37,1^{\prime \prime} \\
\end{array}$ & $\begin{array}{c}\text { N: } 01^{\circ} \\
41^{\prime \prime} 58,8^{\prime \prime} \\
\text { W: } \\
11260^{\circ} \\
11^{\prime \prime} 26,7^{\prime \prime}\end{array}$ & 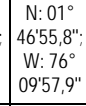 & $\begin{array}{c}\text { N: } 01^{\circ} \\
46^{\prime} 55,8^{\prime \prime} \\
\text { W: } 76^{\prime} \\
09^{\prime} 57,9^{\prime \prime} \\
\end{array}$ & $\mid \begin{array}{c}\text { N: } 01^{\circ} \\
47^{2} 23,2^{\prime \prime} \\
\text { W: } \\
09^{\prime} 170^{\circ} \\
0^{\prime \prime},\end{array}$ & $\begin{array}{c}\text { N: }: 1^{\circ} \\
56^{2} 24,8^{\prime \prime} ; \\
\text { W: } \\
45^{\circ} \\
42^{\circ} 45,4^{\prime \prime} \\
\end{array}$ & $\begin{array}{c}\text { N: } 01^{\circ} \\
56^{0} 06,0^{\prime \prime} \\
\text { W: } \\
45^{\circ} 47,1^{\prime \prime} \\
42^{2} 4,1^{\prime \prime}\end{array}$ & $\begin{array}{c}\mathrm{N}: 02^{\circ} \\
122^{\prime \prime} 53,3^{\prime \prime} \\
\text { W: } \\
55^{\circ} 34,4^{\prime \prime} \\
\end{array}$ & $\begin{array}{c}\text { N: } 02^{\circ} \\
44^{\prime \prime} 47,9^{\prime \prime} \\
\text { W: } 5^{\circ} \\
12^{\prime} 37,1^{\prime \prime} \\
\end{array}$ & $\left\{\begin{array}{l}\text { N: } 02^{\circ} \\
44^{4} 54,2^{\prime \prime} \\
\text { w: } 75^{\circ} \\
1244,5^{\prime \prime}\end{array}\right.$ & \begin{tabular}{|} 
N: $01^{\circ}$ \\
$45: 57,7^{\prime \prime}$ \\
W: $76^{\circ}$ \\
$12 ' 19,0^{\prime \prime}$
\end{tabular} & 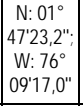 & $\begin{array}{c}\text { N: } 01^{\circ} \\
41^{\prime} 58,8^{\prime \prime} \\
\text { W: } \\
11^{\prime} 26,7^{\prime \prime} \\
\end{array}$ & \begin{tabular}{|c|} 
N: $01^{\circ}$ \\
$5606,0^{\prime \prime}$ \\
W: $70^{\circ}$ \\
$42^{\prime} 47,1^{\prime \prime}$
\end{tabular} & $\begin{array}{c}\mathrm{N}: 01^{\circ} \\
55^{\prime} 06,9^{\prime \prime} \\
\text { W: } \\
45^{\circ} \\
41^{\circ} 48,9^{\prime \prime}\end{array}$ & $\begin{array}{c}\text { N: } 01^{\circ} \\
56^{2} 24,8^{\prime \prime} \\
\text { W: } \\
45^{\circ} 45,4^{\prime \prime} \\
\end{array}$ & \begin{tabular}{|c|}
$\mathrm{N}: 02^{\circ}$ \\
$133^{\prime 2} 2,0^{\prime \prime}$ \\
W: \\
$55^{\circ} 04,3^{\circ}$
\end{tabular} & $\begin{array}{c}\mathrm{N}: 02^{\circ} \\
1830,6^{\prime \prime} \\
\text { W: } 75^{\circ} \\
48^{\prime} 08,6^{\prime \prime}\end{array}$ & $\begin{array}{c}\mathbf{N}: 02^{\circ} \\
1253,3^{\prime \prime} \\
\text { W: } \\
50^{\prime} 34,4^{\circ \prime \prime} \\
\end{array}$ & \begin{tabular}{|c} 
N: 02 \\
$44^{\circ} 47,9^{\prime \prime}$ \\
W: $75^{\circ}$ \\
$122^{\prime} 37,1^{\prime \prime}$
\end{tabular} & INA \\
\hline Altitude & 1766 & 1766 & 1396 & 1213 & 1307 & 1307 & 1197 & 1218 & 1218 & 1197 & 1766 & 1423 & 1423 & 1396 & 1213 & 1307 & 1116 & 1197 & 1218 & 1572 & 1396 & 1766 & 1307 & 1809 & 1213 & 1120 & 1321 & 1116 & 1197 & 1218 \\
\hline $\begin{array}{c}\text { Sample } \\
\text { Element }\end{array}$ & 1.1- HA & 1.2- $\mathrm{HC}$ & 1.3-HB & 2.1- $\mathrm{HA}$ & 2.2- HB & 2.3-HB & 4.1- $\mathrm{HB}$ & 4.2- $\mathrm{HB} \mid$ & 4.3-HC & 4.4. $\mathrm{HC}$ & 1.4 & 1.5 & 1.6 & 1.7 & 2.4 & 2.5 & 3.1 & 4.5 & 4.6 & 1.8 & 1.9 & 1.10 & 2.6 & 2.7 & 2.8 & 3.2 & 3.3 & 3.4 & 4.7 & 4.8 \\
\hline $\mathrm{Na} 2 \mathrm{O}$ & 1 & 2.3 & 1.5 & 2.5 & 2.7 & 2.8 & 2.9 & 2.3 & 2.5 & 2.5 & 2.2 & 2.7 & 3.2 & 2.9 & 0.5 & 0.7 & 2.9 & 3.4 & 3.4 & 3.5 & 3.8 & 3.9 & 3.5 & 2.1 & 3.1 & 3.1 & 2.1 & 3.5 & 3.7 & 3.4 \\
\hline $\mathrm{MgO}$ & 0.8 & 0.4 & 1 & 2.1 & 2.3 & 2.1 & 2.5 & 1.2 & 1.1 & 2.7 & 0.5 & 0.7 & 0.5 & 0.6 & 2.1 & 2 & 1.1 & 0.7 & 0.8 & 1 & 0.9 & 1.5 & 1.7 & 2.1 & 1.5 & 1.5 & 0.5 & 1.4 & 2.1 & 1.4 \\
\hline $\mathrm{Al} 2 \mathrm{O} 3$ & 13.5 & 18.3 & 15.9 & 13.1 & 14.8 & 14.1 & 16 & 16.2 & 15.2 & 15.6 & 13.8 & 12.7 & 13.9 & 14.2 & 15.8 & 12 & 14.1 & 13.8 & 13.7 & 14.5 & 13.9 & 15.5 & 14.4 & 13.6 & 14.3 & 13.3 & 16.3 & 14.3 & 13.8 & 13.2 \\
\hline $\mathrm{SiO} 2$ & 67.6 & 66 & 63.7 & 60.6 & 60.5 & 64.8 & 57.3 & 65.1 & 67.3 & 57.3 & 73.9 & 74.5 & 72.5 & 71.8 & 58 & 66.6 & 70.8 & 72.1 & 72.4 & 71.5 & 71.5 & 66.5 & 67.8 & 65.8 & 64.7 & 68.9 & 70.7 & 66.7 & 68.4 & 71.4 \\
\hline K2O & 2.8 & 4.5 & 2.7 & 2.3 & 1.7 & 2.4 & 1.7 & 2.7 & 3.2 & 1.7 & 4.1 & 3.2 & 3.9 & 3.5 & 2.1 & 3 & 3.3 & 4.2 & 4.2 & 2.6 & 3.2 & 3.5 & 2.9 & 2.6 & 2.8 & 2.9 & 3.7 & 3 & 2.8 & 3.3 \\
\hline $\mathrm{CaO}$ & 0.9 & 0.3 & 1.1 & 4.6 & 4.9 & 3.6 & 5.4 & 2.6 & 2.4 & 6.5 & 0.6 & 1.1 & 1.1 & 1.2 & 1.7 & 1.6 & 1.4 & 1.9 & 1.9 & 1.5 & 1.7 & 1.7 & 3.2 & 3.6 & 4.1 & 2.4 & 0.2 & 3.3 & 2.7 & 2.6 \\
\hline TiO2 & 0.7 & 0.4 & 0.6 & 0.6 & 0.8 & 0.6 & 1.2 & 0.7 & 0.5 & 1.3 & 0.4 & 0.4 & 0.4 & 0.4 & 0.9 & 0.8 & 0.4 & 0.3 & 0.2 & 0.4 & 0.4 & 0.6 & 0.5 & 0.5 & 0.9 & 0.6 & 0.4 & 0.5 & 0.5 & 0.4 \\
\hline $\mathrm{MnO}$ & 0.1 & 0.1 & 0.1 & 0.1 & 0.1 & 0.1 & 0.1 & 0.1 & 0.1 & 0.2 & 0.1 & 0.1 & 0.1 & 0.1 & 0.2 & 0.1 & $<0.1$ & 0.1 & $<0.1$ & 0.1 & 0.1 & 0.1 & 0.1 & 0.1 & 0.1 & 0.1 & 0.1 & 0.1 & 0.1 & 0.1 \\
\hline $\mathrm{Fe} 2 \mathrm{O} 3$ & 3.7 & 2.3 & 5.7 & 5.3 & 7.7 & 5.8 & 8.4 & 4.3 & 3.7 & 8.5 & 2.1 & 3.1 & 2.6 & 2.8 & 11.7 & 8.9 & 3.6 & 2.2 & 2 & 3.2 & 3.1 & 4.4 & 4.7 & 7.6 & 6.8 & 5.3 & 3.5 & 4.1 & 4 & 3 \\
\hline $\mathrm{ZnO}$ & $<0.1$ & $<0.1$ & $<0.1$ & $<0.1$ & $<0.1$ & $<0.1$ & $<0.1$ & $<0.1$ & $<0.1$ & $<0.1$ & $<0.1$ & $<0.1$ & $<0.1$ & $<0.1$ & $<0.1$ & $<0.1$ & $<0.1$ & $<0.1$ & $<0.1$ & $<0.1$ & $<0.1$ & $<0.1$ & $<0.1$ & $<0.1$ & $<0.1$ & $<0.1$ & $<0.1$ & $<0.1$ & $<0.1$ & $<0.1$ \\
\hline $\mathrm{Rb} 2 \mathrm{O}$ & $<0.1$ & $<0.1$ & $<0.1$ & $<0.1$ & $<0.1$ & $<0.1$ & $<0.1$ & $<0.1$ & $<0.1$ & $<0.1$ & $<0.1$ & $<0.1$ & $<0.1$ & $<0.1$ & $<0.1$ & $<0.1$ & $<0.1$ & $<0.1$ & $<0.1$ & $<0.1$ & $<0.1$ & $<0.1$ & $<0.1$ & $<0.1$ & $<0.1$ & $<0.1$ & $<0.1$ & $<0.1$ & $<0.1$ & $<0.1$ \\
\hline $\mathrm{SrO}$ & $<0.1$ & $<0.1$ & $<0.1$ & 0.1 & 0.1 & $<0.1$ & 0.1 & 0.1 & $<0.1$ & 0.1 & $<0.1$ & $<0.1$ & $<0.1$ & $<0.1$ & $<0.1$ & $<0.1$ & $<0.1$ & $<0.1$ & $<0.1$ & $<0.1$ & $<0.1$ & $<0.1$ & $<0.1$ & $<0.1$ & $<0.1$ & $<0.1$ & $<0.1$ & $<0.1$ & $<0.1$ & $<0.1$ \\
\hline $\mathrm{ZrO2}$ & 0.1 & $<0.1$ & $<0.1$ & - & - & $<0.1$ & $<0.1$ & 0 & $<0.1$ & $<0.1$ & $<0.1$ & $<0.1$ & $<0.1$ & $<0.1$ & $<0.1$ & $<0.1$ & $<0.1$ & $<0.1$ & $<0.1$ & $<0.1$ & $<0.1$ & $<0.1$ & $<0.1$ & $<0.1$ & 0.1 & $<0.1$ & $<0.1$ & $<0.1$ & $<0.1$ & $<0.1$ \\
\hline $\mathrm{BaO}$ & 0.1 & 0.1 & 0.1 & 0.1 & 0 & 0.1 & $<0.1$ & 0.1 & 0.1 & 0.1 & 0.1 & 0.1 & 0.2 & 0.1 & 0.1 & 0.1 & 0.1 & 0.1 & 0.1 & 0.1 & 0.1 & 0.1 & $<0.1$ & 0.1 & 0.1 & 0.1 & $<0.1$ & $<0.1$ & 0.1 & 0.1 \\
\hline $\mathrm{PbO}$ & - & $<0.1$ & $<0.1$ & - & - & - & - & - & - & - & - & - & . & . & - & - & $<0.1$ & - & . & 0 & 0 & - & - & - & - & $<0.1$ & - & - & - & - \\
\hline LOI & 8.3 & 5.2 & 7.1 & 8.3 & 3.8 & 3.2 & 3.4 & 4.4 & 3.5 & 2.7 & 2 & 1.4 & 1.5 & 2.1 & 6.3 & 3.7 & 1.9 & 1 & 0.9 & 1.4 & 1.1 & 1.9 & 1 & 1.6 & 0.8 & 1.2 & 2.5 & 2.7 & 1.4 & 0.8 \\
\hline $\mathrm{Cl}$ & $<0.1$ & $<0.1$ & - & $<0.1$ & $<0.1$ & $<0.1$ & $<0.1$ & $<0.1$ & $<0.1$ & $<0.1$ & $<0.1$ & $<0.1$ & $<0.1$ & $<0.1$ & $<0.1$ & $<0.1$ & $<0.1$ & $<0.1$ & $<0.1$ & - & $<0.1$ & $<0.1$ & 0.1 & $<0.1$ & 0.1 & $<0.1$ & $<0.1$ & $<0.1$ & $<0.1$ & $<0.1$ \\
\hline V2O5 & - & - & - & - & - & - & - & - & - & - & - & - & - & - & $<0.1$ & $<0.1$ & - & - & - & - & - & - & - & - & - & - & - & - & - & - \\
\hline $\mathrm{Cr} 2 \mathrm{O} 3$ & - & $\cdot$ & $\cdot$ & - & $\cdot$ & - & $\cdot$ & - & - & - & - & $\cdot$ & - & - & $<0.1$ & $<0.1$ & - & $\cdot$ & - & - & $\cdot$ & - & - & - & - & $\cdot$ & - & - & - & - \\
\hline $\mathrm{NiO}$ & - & - & - & - & - & - & - & - & - & - & - & - & - & - & $<0.1$ & - & - & - & - & - & - & - & - & - & - & - & - & - & - & - \\
\hline
\end{tabular}


Exploratory mineralogical and geomorphology analysis.

\section{- Pitalito}

Guachicos River (formed by El Cedro and Guachicos creeks) supplies water to Pitalito's urban drinking water system. Our observations and samples collected in the field, allowed the geomorphological characterization of the micro-basin of Guachicos River, which has a mountain landscape and a topographical relief of fans and hills. It was also observed that the basin is highly intervened with crops and grazing areas very close to the riverbed. Given the geological characterization of the soil, we concluded that they evolved from fluvial-glacial deposits with influence of volcanic ashes.

Based on the classification proposed by Velandia et al (2001), we identified four geological formations in the micro-basin of Guachicos River: 1) Alluvial processes, terraces, colluviums, fluvial-lacustrine deposits, mud influx, recent alluvial fans from the late Quaternary period; 2) granitoid of Sombrerillos from the Jurassic period, 3) Saldaña formation, tuffs made from volcanic ashes and lapilli, agglomerates and lava, intercalated lithic arenites, litholites and limestones, andesite-dacite porphyry from the Jurassic period and 4) accumulation of granadillo, mudstone and limestone from the Paleozoic period.

In the lower part of the watershed, where El Cedro and Guachicos creeks merge to form the Guachicos River, we found that the fluoride content was $0.381 \pm 0.002$ $\mathrm{mg} / \mathrm{L}$. The concentration was found to be lower in the samples taken from the water system’s checkpoints, down to $0.129 \pm 0.010 \mathrm{mg} / \mathrm{L}$.

\section{- Altamira}

The micro basin that supplies water to the Altamira municipality receives water from three streams: La Perica, La Cerinda, and El Cedro. Water is taken from the latter to supply the local water treatment plant. During our visit to the area, we noticed a negative environmental impact in the upper part of El Cedro basin, probably due to uncontrolled expansion of the agricultural frontier. La Perica creek exhibits a mountainous landscape, full of hills and escarpments. By the geological characterization, we infer that the soils of this basin evolved from igneousmetamorphic rock debris. Nevertheless, due to transitions to a hilly landscape, changes in the geomorphological conditions take place on El Cedro creek, after La Perica and La Cerinda streams merge. We also noticed that soils developed from conglomeratic sandstones, alternating with shales and arcillolites. 
We identified the following geological formations: a) deposits of alluvial processes, terraces, colluviums, mudflows, quaternary deposits; and b) Guapotón soils, migmatites, granulites, granites, quartzite and marbles from the preCambrian period (Velandia et al, 2001).

On La Perica creek, we found a fluoride concentration of $0.199 \pm 0.004 \mathrm{mg} / \mathrm{L}$. The concentration of samples taken from the urban water supply system was similar to the one found on that stream, with a value of $0.197 \pm 0.003 \mathrm{mg} / \mathrm{L}$.

\section{- El Agrado}

La Mosca micro-basin is formed by El Agrado and El Pescado creeks. This basin is characterized by a hilly landscape. For this basin, a geomorphologic analysis shows that soils evolved from conglomerated sandstones and alternating sedimentary materials with shales and arcillolites.

Based on field data, we identified the following geological formations: a) deposits of alluvial processes, terraces, colluvium, mudflows, quaternary deposits; b) Honda group formed by sandstones with intercalations of arcillolites and polymictic conglomerates of the Neogene period; c) Doima formation, which has layers of conglomerates with intercalations of sandstones, limolites and arcillolites of the Placogenic period; d) monzodiorite from the Jurassic period and the Saldaña formation, consisting of ash, agglomerates and lavas, with occasional intercalations of lytic sandstones, limolites, limestones, and Jurassic andesitic porphyries (Velandia et al, 2001).

Despite the difficult conditions of the terrain, this basin exhibited moderate to high human intervention, mainly due to coffee crops on the upper side, impacting downstream hydrology.

The lowest fluoride concentrations were found on La Mosca creek, with values of $0.027 \pm 0.000 \mathrm{mg} / \mathrm{L}$. Drinking water exhibited fluoride concentrations of $0.218 \pm$ $0.005 \mathrm{mg} / \mathrm{L}$.

\section{- Rivera}

Negro river supplies water to the drinking water supply system of the Rivera municipality. Some areas of the basin are well preserved; however, agricultural activities with intense use of pesticides near the riverbed continue to increase. 
Negro river geomorphology is characterized as a hilly landscape. The soils of this basin have developed from conglomeratic sandstones, alternated with shales and arcillolites. The geological formations identified in the Negro river basin are: a) alluvial deposits, terraces, colluvium, fluvial deposits, mudflows from the Quaternary period; b) monzogranite of Algeciras from the Jurassic period (Velandia et al, 2001).

A fluoride concentration of $0.659 \pm 0.009 \mathrm{mg} / \mathrm{L}$ was measured at the water treatment plan inlet tank, reaching values of $0.925 \pm 0.000 \mathrm{mg} / \mathrm{L}$ at checkpoints, turning into the highest levels among the four municipalities.

Prevalence of enamel fluorosis in children from the four Huila municipalities

A total of 840 children between 3-14 years of age were clinically examined using the TF Index (Table 3). The prevalence of fluorosis was 98.2\%. In the four municipalities, the most frequent severity was moderate (70\%). Severe fluorosis was evident in approximately one fifth of the children in each municipality: Altamira (29.1\%), Rivera (24.1\%), Pitalito (21.3\%), and El Agrado (16.4\%) (Figure 2).

Table 3. Prevalence of enamel fluorosis in children from the four Huila municipalities $(\mathrm{n}=840)$.

\begin{tabular}{llll}
\hline \multirow{2}{*}{ Municipality } & \multicolumn{2}{l}{$\begin{array}{l}\text { Presence of } \\
\text { fluorosis }\end{array}$} & $\begin{array}{l}\text { Total number of } \\
\text { children examined }\end{array}$ \\
\cline { 2 - 4 } & No & Yes & \\
\hline Pitalito & 9 & 374 & 383 \\
Altamira & 1 & 103 & 104 \\
El Agrado & 4 & 153 & 157 \\
Rivera & 1 & 195 & 196 \\
Total & 15 & 825 & 840 \\
\hline
\end{tabular}

Figure 2. Enamel fluorosis severity in children from each of the four Huila municipalities.

When examining fluorosis severity by age group, mild fluorosis was most common 
among preschoolers, while severe fluorosis was most common among schoolchildren (Figure 3).

Figure 3. Enamel fluorosis severity by age group in children from each of the four Huila municipalities.

Water Ingestion Exposure Dose (IDag)

The water ingestion exposure doses from checkpoint samples were found to be lower than the recommended total optimal daily dose $(<0.05 \mathrm{mg} / \mathrm{kg} /$ day $)$ for all ages from 6 months (ATSDR, 2003) in the four municipalities (Table 4).

Table 4. Average fluoride water ingestion exposure dose (IDag) from water-supply checkpoints in the four municipalities by age group.

\begin{tabular}{|c|c|c|c|}
\hline Municipality & $\begin{array}{c}\text { Fluoride concentration } \\
(\mathrm{mg} / \mathrm{L}) \\
\text { SD) }\end{array}$ & $\begin{array}{c}\text { Preschoolers IDag } \\
\text { (mg/kg/day) (Mean } \\
\pm \text { SD) }\end{array}$ & $\begin{array}{c}\text { Schoolchildren IDag } \\
\text { (mg/kg/day) (Mean } \pm \text { SD) }\end{array}$ \\
\hline Pitalito & $0.264 \pm 0.213$ & $0.013 \pm 0.011$ & $0.007 \pm 0.005$ \\
\hline Altamira & $0.191 \pm 0.009$ & $0.010 \pm 0.000$ & $0.005 \pm 0.000$ \\
\hline El Agrado & $0.145 \pm 0.221$ & $0.007 \pm 0.011$ & $0.004 \pm 0.006$ \\
\hline Rivera & $0.772 \pm 0.120$ & $0.039 \pm 0.006$ & $0.019 \pm 0.003$ \\
\hline
\end{tabular}

As expected, there was a strong, statistically significant correlation ( $\mathrm{r}=0.986$, $\mathrm{p}<0.05$ ) between average fluoride concentration in drinking water and average fluoride water ingestion exposure dose in preschoolers and schoolchildren (Table 5).

Table 5. Correlation between average fluoride concentration in drinking water and average fluoride water ingestion exposure dose by age group.

\begin{tabular}{lccc}
\hline Age group & $\begin{array}{l}\text { Correlation } \\
\text { coeficient }(\boldsymbol{r})\end{array}$ & $\begin{array}{l}\text { Coefficient of } \\
\text { determination }\left(\boldsymbol{R}^{\mathbf{2}}\right)\end{array}$ & ANOVA \\
\hline Preschoolers & 0.986 & 0.971 & $\mathrm{P}<0.05$ \\
Schoolchildren & 0.935 & 0.874 & $\mathrm{P}>0.05$ \\
\hline
\end{tabular}


Fluoride concentration of table salt

The average fluoride concentration of table salt collected in the four municipalities was under the lower limit (180-220 $\mu \mathrm{g} / \mathrm{g})$ established by the Colombian regulation (Ministerio de Salud, 1996) (Table 6). Since we were not able to determine the daily dose of table salt consumption, that exposure dose was not determined.

Table 6. Average fluoride concentration ( $\mu \mathrm{g} / \mathrm{g}$ ) in table salt samples from the four sentinel municipalities

\begin{tabular}{lcc}
\hline Municipality & \multicolumn{2}{c}{$\begin{array}{c}\text { Fluoride } \\
\text { concentration ( } \mu \text { g/g) } \\
\text { (Mean } \pm \text { SD) }\end{array}$} \\
\hline Pitalito & 158.920 & 16.570 \\
Altamira & 171.850 & 21.556 \\
El Agrado & 167.310 & 15.226 \\
Rivera & 165.050 & 18.270 \\
\hline
\end{tabular}

\section{Discussion}

The department of Huila (Colombia) has a high mineral and geological diversity due to the accumulation of materials from different events and geologic eras. In the analyzed samples, we found the following sediments: sandstones, claystone, conglomerates, materials of volcanic origin like ash and lapilli tuffs, lavas and agglomerates, granite, andesite and dacite materials, migmatites, granulites, neises, granites, quartzite, marbles and intercalated materials deposited by different processes. The Secretary of Mining and Agriculture from the department of Huila (2008) recognizes that there is an active exploitation of phosphoric rock and another (though less significant) exploitation of barite. These activities are responsible for $37 \%$ of the nation's production and presumed to be a strong indicator of fluoride presence in the studied zone.

As these municipalities are characterized by endemic fluorosis we were expecting that this could be currently disclosed in high levels of fluoride from different sources and thus be correlated. Usually only the fluoride levels in tap water from the aqueduct are reported. In this study we decided to further analyze the fluoride 
content in tap water, water from different tributaries, table salt, and soil, rocks and active sediment. We took samples in both the rain and the dry season. We were expecting to have higher fluoride levels then reported. The fluoride analyses performed for the four sentinel municipalities for all soil, rocks and active sediment samples indicated that the fluoride concentrations were lower than 15 $\mathrm{mg} / \mathrm{kg}$. This level was further confirmed by geochemical analysis by X-ray fluorescence (where there was a lack of evidence of fluorine pure minerals) (Table 4). Chandi et al (2015) found the geochemical composition of the rocks to be only responsible for groundwater contamination. The results of the current study suggest that the delivery of fluoride to the watersheds is very unlikely, since normal fluoride concentrations in soil are within the range of 150 to $400 \mathrm{mg} / \mathrm{kg}$ (Blagojevich et al, 2002; Begum, 2012), and the concentrations found in this study were much lower. However, we observed that an external source of fluoride might be the fluoride-containing fertilizers that are being used for agricultural production within the area (Bombik, 2011).

In addition, for the municipalities of Pitalito, Altamira, El Agrado and Rivera, the water current ingestion exposure dose did not exceed the recommended doses and thus a relationship between fluoride-dose and the high prevalence and severity of the developmental defect of enamel, namely enamel fluorosis could not be established.

Conversely, Antonijevic et al (2015) found $11 \%$ of water samples with a fluoride concentration exceeding $1.5 \mathrm{mg} / \mathrm{L}$ and a correlation to fluorosis in schoolchildren. In this study, however, the hypothesis of cumulative exposure to fluoride in water and fluoridated table salt as a possible cause of enamel fluorosis cannot be discarded. It might be possible that fluoride exposure from water took place in conditions different to the ones found in this study and that children could have been exposed to toxic concentrations of fluoride during tooth development. Fluoride concentration from table salt in these municipalities was lower than national norm in all cases. This finding differed from a previous fluoride intake study in young children from four Colombian cities using the duplicate plate method where $86 \%$ of the samples were within or below the regulated fluoride concentration range (Franco et al, 2003), and to the findings by Ramirez et al. (2006) using a questionnaire, where $21 \%$ of samples were above the norm.

If analyses of the current situation of geological factors, water, and concentration of fluoride in table salt did not show association with the prevalence of dental fluorosis found in these children, other risks factors need to be considered. For example, excessive salt consumption. The National Nutritional Status Survey in 
Colombia reported from gastric cancer studies that in Colombia there is a high salt consumption (República de Colombia, 2009). From local studies of fluorosis, which considered risk factors based on questionnaires, a related high consumption of salt was found (Martignon et al., 2002; Arrieta-Vergara et al, 2009). But in the fluoride intake study in Colombia it was found that food accounted for only $24 \%$ of total fluoride intake, beverages to 6\%, while fluoridated toothpaste (1000-1500 ppm) accounted for $70 \%$ of total fluoride intake (Franco et al., 2005). Ingestion of fluoridated toothpaste in young children has been associated to the presence of fluorosis by means of questionnaire (Martignon et al, 2002; Arrieta-Vergara et al, 2009; Misnaza-Castrillón, 2009). In the related-to-this epidemiological study preliminary results assessing knowledge, attitude and practices with a questionnaire to children parents show that parents of most children (70\%) presenting with no dental fluorosis considered that the purpose of the fluoridated toothpaste is the prevention of caries vs. having white teeth, fresh breath, others or not knowing (Martignon et al., 2016). Other relevant aspect is that more than half (56\%) of parents reported that their children swallowed the toothpaste in the first five years of life. But this cannot explain the prevalence of fluorosis higher than $90 \%$ in the examined population. Malnutrition has also been associated on questionnaires to the presence of fluorosis (Ramos-Martínez et al, 2010), but in the simultaneously developed epidemiological study conducted in preschool and schoolchildren in the same four municipalities, where $42 \%$ in each group were classified outside normal nutritional status, no statistical significant association between this finding and the presence of fluorosis was found (Martignon et al., 2016). Mapping the calcium/fluoride ratio may also need to be analyzed to further understand the use of water/salt-fluoridation for caries control (de Souza et al, 2013). Taking into account the costs of the geological analyses, as there is a seasonal variation in the chemistry of water (Jayaprakash et al, 2008), a higher number of geological samplings during the year could also allow for more robust data.

This study can be useful as a database for future assessments related to dental fluorosis presence in children whose teeth were in development at the moment of sampling of fluoride content in geological elements, water, and table salt. We recommend the implementation of a monitoring plan in these municipalities. The plan should consider the review and evaluation of different actions, plans and programs that have been or are to be implemented in the region. Furthermore, the focus of future studies should be on studies combining the daily ingestion of fluoridated table salt, oral hygiene habits, and the analysis of fluoride concentration in the diet, and geological factors, including fertilizers that are being used for 
agricultural production within the area, and conduction of geological samplings in different seasons during the year.

\section{Conclusions}

Geochemical analysis in the municipalities of Pitalito, Altamira, El Agrado and Rivera in the department of Huila (Colombia) revealed no high fluoride concentrations. The concentration of fluoride in table salt was found to be under the inferior limit established by the Colombian regulations and in water intake it did not exceed recommended total dose. The fluoride content of these elements did not show association with the current prevalence of enamel fluorosis in preschool and schoolchildren. Further studies considering combining different direct and indirect fluoride intake sources are recomended.

\section{References}

Alcaldía de Altamira. (2016). Proyecto de acuerdo No. 008 de 2016. Resource document. http://altamira-huila.gov.co/apc-aafiles/35613136323432666237626464323663/acuerdo-008-del-2016-plan-dedesarrollo-sancionado-con-firmas.pdf. Accessed 10 September 2016.

Alcaldía de El Agrado. (2016). Acuerdo No. 015 De 2016. Resource document. http://elagrado-huila.gov.co/apc-aafiles/31616533383962393137653031626163/plan-de-desarrollo-territorial-elagrado-2016-2019.pdf. Accessed 10 September 2016.

Alcaldía de Pitalito. (2016). Acuerdo No. 022 de 2016. Resource document. http://pitalito-huila.gov.co/apc-aa-

files/35333563363431646635326162376136/acuerdo_022-2016.pdf. Accessed 10 September 2016.

Alcaldía de Rivera. (2016). Acuerdo municipal PDM 2016. Resource document. http://www.rivera-

huila.gov.co/Transparencia/Normatividad/Acuerdo\%20Municipal\%20PDM.pdfww w.rivera-huila.gov.co/apc-aa-files/.../pdt-rivera-25-de-marzo-2019.ok.pdf.

Accessed 10 September 2016. 
Antonijevic, E., Mandinic, Z., Curcic, M., Djukic-Cosic, D., Milicevic, N., Ivanovic, et al. (2015). "Borderline" fluorotic region in Serbia: correlations among fluoride in drinking water, biomarkers of exposure and dental fluorosis in schoolchildren. Environmental Geochemistry and Health, 38(3), 885-896.

APHA (2012). Standard methods for the examination of water and wastewater. Washington, DC: Rice, E.W., Baird, R.B., Eaton, A.D., Clesceri. L.S. (Eds.).

Arrieta-Vergara K., González-Martínez F., Luna-Ricardo, L. (2011). Exploración del riesgo para fluorosis dental en niños de las clínicas odontológicas Universidad de Cartagena (Exploration of dental fluorosis risk in children at dental clinics University of Cartagena). Revista de Salud Pública, 13(4), 672-683.

ATSDR (2003). Toxicological profile for fluorides, hydrogen fluoride, and fluorine. Resource document. https://www.atsdr.cdc.gov/ToxProfiles/tp11.pdf. Accessed 19 October 2016.

ATSDR. (2005). Public Health Assessment Guidance Manual (Update). Resource document https://www.atsdr.cdc.gov/hac/phamanual/pdfs/phagm_final1-27-05.pdf. Accessed 20 October 2016.

Begum, A., (2012). Soil Profiles and Fluoride Adsorption in Intensely Cultivated Areas of Mysore District, Karnataka, India. Chemical Science Transactions, 1(2), 410-414.

Blagojevic, S., Jakovljevic, M., Radulovic, M. (2002). Content of fluorine in soils in the vicinity of aluminium plant in Podgorica. Journal of Agricultural Science, 47(1), 1-8.

Bombik, E., Bombik, A., Gorski, K., Saba, L., Bombik, T., Rymuza, K., (2011). The Effect of Environmental Contamination by Fluoride compounds on selected horse tissues. Polish Journal of Environmental Studies, 20(1), 37-43.

Clavijo, J., Ballesteros, C. (1987). Fluorita, in: Recursos Minerales de Colombia (Fluorite, in: Mineral of Colombia). Publicaciones Geológicas Especiales del INGEOMINAS, No. 1 (pp. 839-955). Colombia.

de Souza C., Lima J., Adriano M., de Carvalho F., Forte F., de Farias-Oliveira R., et al. (2013). Assessment of groundwater quality in a region of endemic fluorosis in the northeast of Brazil. Environmental Monitoring and Assessment, 185(6), 4735-4743. 
Fejerskov, O., Larsen, M., Richards, A., Baelum, V. (1994). Dental tissue effects of fluoride. Advances in Dental Research, 8(1), 15-31.

Franco, A., Saldarriaga A., González, M., Martignon S., Arbeláez, M., Ocampo, A., et al. (2003). Concentración de flúor en la sal de cocina en cuatro ciudades colombianas (Fluorine concentration in table salt in four Colombian cities). Revista CES Odontología, 16, 21-26.

Franco A., Martignon S., Saldarriaga A., González M., Arbeláez M., Ocampo A., et al. (2005). Total Fluoride Intake in Children aged 22-35 Months in Four Colombian Cities. Community Dentistry and Oral Epidemiology, 33(1), 1-8.

Frankenberg, W., Tabatabai, M., Adriano, D., Doner, H. (1996). Bromine, Chlorine and Fluorine, in: Methods of Soil Analysis Part 3-Chemical Methods, SSSA Book Ser. 5.3., Sparks, D.L., Page, A.L., Helmke, P.A., Loeppert, R.H. (Eds.), Madison, (pp. 833-867), doi:10.2136/sssabookser5.3.c31.

ICONTEC, 1995. Norma Técnica Colombiana NTC/ISO 5667-1. Norma Técnica Colombiana NTC/ISO 5667-1. Gestión ambiental. Calidad del agua. Muestreo. Directrices para el diseño de programas de muestreo. (Colombian technical rule NTC/ISO 5667-1 environmental management. Water quality. Sampling. Guidelines for the design of sampling) Bogotá. Resource document. http://ingenieria.udea.edu.co/isa/normas_decretos/norma\%20muestreo.pdf. Accessed 5 September 2016.

IGAC. (1994). Estudio General de Suelos del Departamento del Huila. Bogotá (General soil study from department of Huila. Bogotá). Resource document. ftp://gisweb.ciat.cgiar.org/dapa/users/apantoja/london/colombia/suelos/00_shape_s uelos/departamentales_2011_brayan_silvia/huila/memoria\%20tecnica/introducci\% c3\%93n\%20tomo\%20i.pdf. Accessed 8 October 2016.

IGAC. (2008). Atlas básico Colombia (Basic Atlas from Colombia). Volume 2, seventh edition. Bogotá: IGAC (Ed.).

Jayaprakash, M., Giridharan, L., Venugopal, T., Krishna Kumar S. P., Periakali P. (2008). Characterization and evaluation of the factors affecting the geochemistry of groundwater in Neyveli, Tamil Nadu, India. Environ Geol, 54(4), 855-867.

Jiménez, G., Moncada, O., López, P., Camargo, A. (2006). Municipios afectados por fluorosis dental en el departamento del Huila soluciones, implementación y seguimiento - informe municipios El Pital y El Agrado (Municipalities affected by 
dental fluorosis department of Huila - solutions, implementation and monitoring El Pital and El Agrado municipalities report). Dirección Nacional de Investigaciones - Universidad Antonio Nariño.

Marín, L., Cury, J., Tenuta, L., Castellanos, J., Martignon, S. (2016). Higher Fluorosis Severity Makes Enamel Less Resistant to Demineralization. Caries Research, 50(4), 407-413.

Martignon, S., Granados-Cepeda. O. (2002). Prevalencia de fluorosis dental y análisis de asociación a factores de riesgo en escolares de Bogotá (Dental fluorosis prevalence and risk factors association analysis in scholar children from Bogotá). Revista Científica Facultad de Odontología Universidad El Bosque, 8, 19-27.

Martignon, S., Castiblanco, G., Opazo, M., Usuga-Vacca, M., Marin, L., SilvaHermida, L., et al. (2016). Colombian-children endemic fluorosis associated with biologic, environmental, and psychosocial aspects. \{Abstract\}. 45thAnnual meeting of the AADR/ $40^{\text {th }}$ annual meeting of the CADR. Los Angeles California.

Ministerio de Salud. (1996). Decreto 547 en cuanto a la expedición del registro Sanitario y a las condiciones sanitarias de producción, empaque y comercialización, al control de la sal para consumo humano y se dictan otras disposiciones sobre la materia. Bogotá (Decree 547, by which the tittle V of the law 09/1979 is regulated as regards the issuance of the sanitary registration and the sanitary conditions of production, packaging and marketing of table salt). Resource document.

https://www.invima.gov.co/images/stories/aliementos/decreto_547_1996.pdf. Accessed 1 October 2016.

Misnaza-Castrillón, S. (2009). Relación de factores de riesgo con la severidad de la fluorosis dental confirmada por clínica, en la población de 5 a 19 años atendida en la IPS indígena y en la ESE del municipio Cuaspud Carlosama, Nariño, Colombia (Relationship between risk factors and severity of dental fluorosis clinically diagnosed, in a 5 to 19 years old population attended in two health service centers for indigenous people from Cuaspud Carlosama municipality, department of Nariño, Colombia). Biweekly national epidemiological report. 14 (19), 289-304.. Informe Quincenal Epidemiológico Nacional. 14(19), 289-304. Resource document.

http://www.ins.gov.co/iqen/IQUEN/IQEN\%20vol\%2014\%202009\%20num\%2019 .pdf. Accessed 3 September 2016. 
Montaña-Sala, M. (2008). Guía de fluorosis dental - Normas técnicas de la fluorosis dental (Dental fluorosis guideline - technical rules). Gobernación del Huila, Secretaría de Salud Departamental. Neiva. Resource document. http://huila.gov.co/documentos/G/guia_fluorosis_dental_huila.pdf. Accessed 10 September 2016.

OPS/OMS. (1995). Manual para la vigilancia epidemiológica de los programas de fluoruración de la sal (Manual for the epidemiological surveillance of salt fluoridation programs). Washington D.C. in Tovar, S., Misnaza, S. (2016). Technical document of fluoride vs caries and dental fluorosis. Bogotá. (C) Ministerio de Salud y Protección Social, versión 2.0 - febrero. Resource document. https://www.minsalud.gov.co/sites/rid/Lists/BibliotecaDigital/RIDE/VS/PP/ENT/p erspectiva-uso-fluor.pdf. Accessed 1 September 2016.

Ramírez, B., Franco, A., Sierra, J., López, R., Alzate, T., Sarrazola, A., et al. (2006). Fluorosis dental en escolares y exploración de factores de riesgo: municipio de Frontino (Dental fluorosis in scholar children and exploration of risk factors: municipality of Frontino) Revista Facultad de Odontología Universidad de Antioquia, 17(2), 26-33.

Ramos-Martínez, K., González-Martínez, F., Luna-Ricardo, L. (2009). Estado de salud oral y nutricional en niños de una institución educativa de Cartagena (Oral health status in children of a educational institution from Cartagena). Revista Salud Pública, 12(6), 950-960.

República de Colombia. (2009). Protocolo de investigación - Encuesta Nacional De La Situación Nutricional En Colombia 2010 (Research protocol - National Nutritional Status Survey in Colombia). Resource document. https://www.minsalud.gov.co/sites/rid/Lists/BibliotecaDigital/RIDE/VS/ED/GCFI/ Base\%20de\%20datos\%20ENSIN\%20-\%20Protocolo\%20Ensin\%202010.pdf. Accessed 28 March 2017.

Rojas, A., Prieto, G., Tabares, L., Garcia, D., Neira, G., Sanchez, C. (2010). Evaluación geoquímica para geología médica en el Departamento de Santander (Geochemical assessment for medical geology in the department of Santander). INGEOMINAS Bogotá. Resource document. https://www.academia.edu/25409730/evaluaci\%c3\%93n_geoqu\%c3\%8dmica_para _geolog\%c3\%8da_m\%c3\%89dica_fluorosis_en_el_departamento_de_santander. Accessed 8 October 2016. 
Secretary of Mining and Agriculture from the department of Huila. (2008). Expectativas del negocio minero en el territorio departamental (Expectations of the mining business in the departmental territory). Resource document. http://www.huila.gov.co/documentos/P/panorama_minero_dptal.pdf. Accessed 11 September 2016.

Servicio Geológico Colombiano. (2011). Fluorosis in Huila Department - final inform. Bogotá. Resource document.

http://aplicaciones1.sgc.gov.co/sicat/html/Metadato.aspx?CID=241359. Accessed 13 August 2016.

Suma R., Shashibhushan K., Shashikiran N., Subba R. (2008). Progression of artificial caries in fluorotic and nonfluorotic enamel: an in vitro study. The Journal of Clinical Pediatric Dentistry. 33, 127-130.

Tellez M., Santamaria R.M., Gomez J., Martignon S. (2012). Dental fluorosis, dental caries, and quality of life factors among schoolchildren in a Colombian fluorotic area. Community Dental Health, 29(1), 95-99.

Thomas, J., Glass, H., White W., Trandell R. (1977). Flouride Content of Clay Minerals and Argillaceous Earth Materials. Clays and Clay Minerals, 25(4), 278284.

Thylstrup, A., Fejerskov, O. (1978). Clinical appearance of dental fluorosis in permanent teeth in relation to histologic changes. Community Dentistry and Oral Epidemiology, 6(6), 315-328.

Velandia, F., Nuñez, A., Marquinez, G. (2001). Memoria explicativa mapa geológico del Departamento del Department of Huila. Explanatory memory of the Department of Huila geological map. INGEOMINAS Technical Report. Bogotá, doi: 10.13140/2.1.3373.0885.

Zinck, J. A. (2012). Geopedología: Elementos de geomorfología para estudios de suelos y de riesgos naturales (Geopedology: geomorphology elements for studies of soils and natural hazards). Faculty of Geo-Information Science and Earth Observation. ITC. The Netherlands. Resource document. "https://www.researchgate.net/publication/259935872_Geopedologia_Elementos_ de_geomorfologia_para_estudios_de_suelos_y_de_riesgos_naturales. Accessed 1 September 2016. 